\title{
An Agent-Based Internet of Things Platform for Distributed Real Time Machine Control
}

\author{
Alberto Rivas, Pablo Chamoso and Sara Rodríguez \\ BISITE Research Group, Department of Computer Science and Automation Control, University of Salamanca \\ Plaza de la Merced s/n, 37008, Salamanca, Spain \\ \{rivis,chamoso,srg\}@usal.es
}

\begin{abstract}
The way in which the Internet of Things and the Web of Things improve everyday objects may seem obvious; elements that make up our daily life are increasingly interconnected and it is becoming more common for us to be surrounded by them. However, the possibilities these technologies offer are not only limited to routinely used objects. By adapting these still emerging technologies, any kind of an object can achieve better performance. They can, for example be applied to research tools, to obtain faster search results and improve the user's experience. The presented work follows these lines; we present a Web-operated machine for the study of the behaviour of certain animals. In addition, the proposed architecture favours the addition of cognitive abilities, due to the inclusion of a Multi-Agent System.
\end{abstract}

Keywords-Multi-Agent Systems, Agents, Internet of Things, Web of Things, Odour Dispensator

\section{INTRODUCTION}

The term, "Internet of Things (IoT)" is commonly understood as the networked interconnection of everyday objects, which are often equipped with ubiquitous intelligence [1]. However, not only daily objects can be interconnected with the currently essential smart devices, IoT makes it possible to connect and communicate all kinds of objects and physical devices. Thus, the IoT technology opens up a lot of new possibilities to using a large number of new applications that improve the quality of some of the aspects of our lives. This is partly due to the great evolution that technology has undergone in recent years, where, in addition to increasing its processing and communication capacity, its size has been reduced. Thus, it is possible to incorporate connectivity capabilities into a great number of objects.

Since an increasing number of devices is getting connected, the next logical step is to use the World Wide Web and its associated technologies as a platform for smart things (ie, sensor and actuator networks, embedded devices, electronic appliances and digitally enhanced everyday objects) [2]. Closely related to IoT, the term Web of Things (WoT) goes beyond the Internet approach; the technologies and the patterns used in the traditional web are leveraged to fully integrate smart things and their services in the Web. Thus, by integrating objects, the end user can easily control the object or obtain knowledge about it from any computer or mobile device.

Communication capabilities are not the only feature of technology that we can benefit from. Its improved processing capacity is also a big advantage and it can be incorporated into objects; this makes it possible to provide objects with certain cognitive or autonomous behaviours which equip them with a level of intelligence when acting or making decisions. MultiAgent Systems (MAS) are able to provide this part of Artificial Intelligence (AI). MAS are groups of agents that are capable of solving problems in a distributed manner by making good use of the agents' social and individual behaviours [3].

Thus, simple objects can act as agents when communicated in a way that permits collaboration for the achievement of a common goal. It is possible, therefore, to have a simple object that behaves as an agent or a complex object, divisible into multiple agents which are connected to one another. Considering the latter example, the present work focuses on a complex machine that is divisible into different interconnected parts.

Specifically, the current work presents an architecture that is capable of providing a solution which interconnects complex objects with technological devices such as computers, smartphones or tablets, enabling us to control them through the Internet and more concretely through the Web. The object with which the study was carried out is used for research in the field of neurosciences for the study of the behaviour of animals in the presence of different odours.

The article first discusses the current state of the art related to the technological side of studying mice behaviour. We then provide a summary of the current state of the technologies used in the present work, in order to understand the hardware and software architecture, which will be explained below. Subsequently, the procedure carried out in the study and the results obtained are described in detail. Finally, the work ends with a conclusions and future lines of work section.

\section{STATE OF THE ART}

\section{A. Internet of Things}

The IoT is one of the major trends in the development of technologies in the Information and Communication Technologies (ICT) [4]. Its purpose is to make different activities easier for the user, in addition to providing new possibilities, which for example, enable easier monitoring. Computing can now be embedded everywhere and programmed to act automatically with no need for manual triggers.

In IoT, the elements that can be found in the environment and that we call things, machines or objects, are improved with 
the use of computing and communication technology. Thus, they become new Internet or network users that generate data traffic [5].

Thanks to this, IoT is able to offer different services based on person-to-person, person-to-machine, machine-to-person or machine-to-machine interactions [6]. These IoT-based services automate some of the activities that before were carried out by people by assigning them to machines; this leads to improvements in the manufacturing industry, in offices, homes and everywhere else.

The communication that links machines with other machines or services [7] can be achieved in many different ways, the most commonly used is the IEEE 802.11 standard which offers wireless connectivity [8].

\section{B. Agents Theory}

One of the main goals of applying IoT and WoT is to convert those objects, things or machines into intelligent devices. To this end, the machine must be given an amount of knowledge that will make it capable of acting autonomously in specific situations.

When modelling human decisions, the main models followed are agent-based models [9]. Extensive literature can be found on agent theory. Since decades, the term agent has been used to describe an autonomous entity capable of interacting with the surrounding environment. MAS are based on individual agents who have common objectives within the system [10] and collaborate together in order to solve complex problems that a unique entity would not be able to solve.

Organizations are one of the main characteristics of MAS. Different definitions of this term have been proposed. A practical proposal was given by Jennings and Wooldridge in [11], where they said that "We view an organisation as a collection of roles, that stand in certain relationships to one another, and that take part in systematic institutionalised patterns of interactions with other roles".

The role, therefore, is one of the key concepts in MAS organizations. It is a description of an abstract behaviour of agents. A role describes the constraints (obligations, requirements, skills) that an agent will have to satisfy to obtain a role, the benefits (abilities, authorization, profits) that an agent will receive in fulfilling that role, and the responsibilities associated to that role [12].

\section{ARChiteCtURE OVERVIEW}

The developed architecture consists of two distinct parts. On the one hand we have the machine hardware, responsible for the operation of the machine and communication with the control and monitoring device used by the user. On the other hand, we have the software, responsible for providing the end user with all available options and the real-time status of the machine, as well as triggering all actions that the actuators must perform, following the logic and the operation of the task which is desired. The software part of the architecture runs in a distributed way, running one part in the client device and another part in the machine hardware.
In this case, by means of decision techniques, the machines involved decide among themselves which one acts as a server machine, executing the MAS, the MQTT Broker and the database. Figure 1 shows a diagram of the architecture for $\mathrm{m}$ machines.

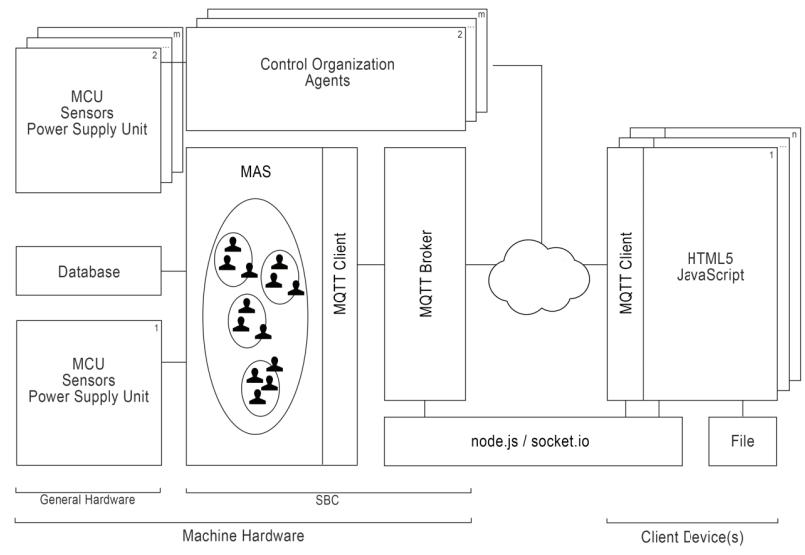

Fig. 1. Multi-Machine Architecture Schema

From the diagram, it can be observed that machine 1 acts as a server, while the other machines are connected through the Network, executing in their corresponding SBCs (Small Board Computer) the software with which their agents are represented. These agents are represented in the MAS by VO groupings.

\section{A. Hardware Description}

Generally, the hardware of the machine or a desired set of machines, is adapted to the outlined scheme. Thus, the hardware set presented in this work, is composed of two well differentiated parts:

- $\quad$ An SBC with a $900 \mathrm{MHz}$ processor and $512 \mathrm{Mb}$ of RAM, it hosts the servers necessary for communication, offering the specific services of the machine that is being treated. In addition, it performs access point functions to link control devices to the system via Wi-Fi. In this $\mathrm{SBC}$, the business logic software is executed, this will be explained later on, as well as monitoring and control software.

- A motherboard that monitors all sensors and acts on the robotic elements of the system, connected directly to the SBC. In this part hardware is executed the firmware that will be detailed in the following section.

Thus, any machine that is to function with the designed platform must have, in all cases, a SBC for control and monitoring and a specifically designed motherboard for the accomplishment of tasks that are to be carried out by that particular machine. 


\section{B. Software Description}

The software platform is subdivided into different parts, where each one is centralized on providing solutions for certain functionality. In addition, it contains a system that is distributed in the firmware, which runs on the hardware of the machine itself. The software that encapsulates business logic runs on a microcomputer which is connected to the hardware part of the machine and the client. Also, monitoring software which runs on the client side, allowing to operate the machine through different devices.

\section{Firmware}

The first part of software is firmware which resides as lowest level software; the way in which the machine is to function is programmed at this level. In terms of execution times, it solves the most critical tasks and manages the way information is obtained from the sensors at all times, communicating the necessary information to the business logic software using a proprietary protocol through serial communication. In the opposite direction of communication, the business logic software sends actions that are to be performed, these actions are dealt with in the order of the established priority.

\section{Business logic software}

This software consists of several parts and tries to provide the best response to the business logic of the machine. That is, it encapsulates knowledge about behaviour and translates it to the actions that need to be carried out in each moment. This is the part that encapsulates most of the MAS functionality.

MAS, based on Virtual Organizations (VO), presents a generic scenario for the control of any type of machine in which it is necessary to manage information, keep a record of what happened and carry out different tasks, understanding task as an independent functionality that together reaches the ultimate goal of the machine.

In this way, there are two VOs: the Support Organization (SO) and the Control Organization. The first, SO, supports the system in terms of access to information and registration of actions, dissociating business logic from the way it is treated and accessing the information. There are three default roles that will be common regardless of the machine you want to control:

- Information Agent: It assumes an access point to the information, allowing the consultant to take care of the implementation.

- Dialog Agent: This agent serves as the communication client, deciding what information is to be transmitted and which is not. In addition, it receives actions from exterior, so it decides what events to end as a consequence.

- $\quad$ Log Agent: This agent is responsible for recording everything that happens in the system, establishing a series of codes and registering previously configured parameters.

On the other hand, in the Control Organization (CO), we have a set of roles that the agents will take on to perform each task (as many agents as necessary for each of the roles). Thus, in the $\mathrm{CO}$, we can find agents that are specialized only in the task they must perform and are isolated from the rest of the system during its execution.

The Norm Agent (NA) is found in both of the organizations. This role is taken on by the agents who are in charge of the security of the system and who regulate the communications within it. For example, they only allow $\mathrm{CO}$ agents to access the information that is relevant to their task.

\section{Monitoring and control software}

The last part of the software is that in charge of controlling and monitoring the machine or machines being used. Therefore, this software is the one used by the expert user in the study to be performed through the machine.

Since each machine requires very different specifications for the expert user from each other, this part will vary depending on the case study.

However, the technologies used share some common characteristics which allow to maintain the machines in a realtime mode and to operate simultaneously for multiple users. These features are achieved in large part due to the use of node.js and socket.io.

\section{RESULTS}

To evaluate the system, a case study has been carried out which intends to examine the behaviour of mice, although it would be applicable to the study of the behaviour of any animal subject, in the field of automated olfactory stimulus dosage. The system that has been developed in this work consists of two main parts: the hardware system and the software system; these are detailed below.

\section{A. Hardware System}

The specific hardware for the present case study is divided into a set of modules that follow the scheme shown in Figure 2.

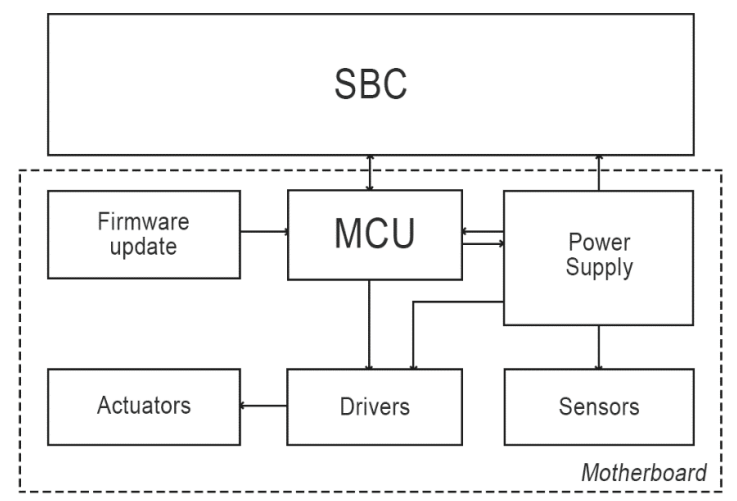

Fig. 2. Specific Hardware Schema

Since the SBC is common in all cases (saving some aspects of configuration and the software that runs on it), the most specific part is the motherboard. It is the main management unit of the mechanical or mobile elements of the odour presentation system. It is composed of a MCU (Microcontroller 
Unit), power drivers which act on the solenoid valves and sensor inputs. The sensors available to the system are:

\section{i. Presence sensor of the study subject \\ ii. Contact sensor of the subject on the liquid dispensing member}

iii. Flow sensor on the two air flows of the system

\section{iv. System temperature sensor}

The MCU (Microcontroller Unit) guarantees precision in the dosing and flow control systems, since the firmware designed for this purpose allows for a comprehensive control of the times. The components used as power drivers for the solenoid valves which control the air flows have a very short response time (approx. 5us), and the response time of the solenoid valves which is approximately $20 \mathrm{~ms}$, is taken into account to trim the times of the different stages of the process.

The presence sensor of the study subject is designed on the basis of cutting an infrared light beam. The trigger has a negligible delay for our object of study and it is attended by interruption in the firmware, which provides immediacy in the recording and processing of the different events of the process.

The contact sensor of the subject on the liquid dispensing element is designed on a capacitive sensor. Previously, a register was made of the contacts produced between the tongue of the subject of study and the sensors through a short circuit. That is, the subject itself served as an electrical conductor which closed the circuit when its tongue and legs touched the two points of the open circuit. Although the electric flow flowing through the subject's body was negligible, it was preferable not to interact electrically with the subjects. In addition, the periods of controlled dehydration to which subjects were exposed prior to testing, greatly reduced their ability to conduct electricity. The capacitive sensor detected small alterations in the capacities of the other sensor (liquid dispensing element) and when these alterations exceeded the threshold, a trip was produced which was treated in the same way as the trip of the presence sensor (IR).

The flow sensors capture the airflow of the two flow rates of the system by sending a proportional and analog signal to the MCU that is converted into a digital signal by the ADC (Analog to Digital Converter). This data is sent to the SBC where a PID (Proportional-Integral-Derivative) control algorithm calculates a control signal that is sent back to the MCU. The control signal is applied by PWM (Pulse-Width Modulation) signal to proportional solenoid valves closing the control loop.

\section{B. Software System}

\section{Firmware}

Firmware is the software that runs on hardware, specifically designed for the odour dispensing machine.

This part is connected bidirectionally to the SBC through a reduced, encrypted and byte-oriented protocol that has been specifically designed to reduce its size and thus the communication time, which is done through port Series at 115200 bps.
When a new study is being performed, the SBC communicates the configuration to the firmware. The firmware will autonomously execute all actions on the basis of this communication. Therefore, when the tasks are being performed, the firmware is self-sufficient and only has to make information available to the Business Logic Software which receives it in the form of notifications.

To obtain the information, different interruptions are generated by the connected sensors. In this way, the firmware is freed from having to sound periodically the sensors in search of a new data, optimizing the hardware processing capacity. According to this scheme, when a sensor changes its state, an interruption occurs that analyses the change providing a new data, which is processed and sent to the SBC.

On the other hand, the firmware translates the information that comes from the Business Logic Software into actions that the actuators have to carry out in a controlled manner and following the logic established in the configuration.

The control of timelines is carried out simultaneously by the firmware itself. Up to five timed events can be controlled with high precision; this has been predetermined by the custom design of this hardware, which uses high stability quartz watches. Such high precision is achieved that it is not necessary to evaluate the correctness of the scientific study.

\section{Business logic software}

Although MAS is used in a generic way and has already been described, it is necessary that we go into more detail on the functioning of a specific MAS part in the current case study, concretely, the variables associated with the CO.

In the case of the present stimulus system, the roles present in the $\mathrm{CO}$ are the following:

- Caudal Agent: Two agents are responsible for fulfilling this role, this is because in the present case there are two flows to control. Its function is to maintain the desirable flow rate that has been previously configured from the Monitoring and Control Software and has been stored by the Information Agent.

- $\quad$ Task Agent: The corresponding agent is in charge of the correct execution of the study and the tasks specified by the user. $\mathrm{He}$ is aware of the restrictions of each test and is able to choose the odours to be delivered according to the given norms. Also, this agent evaluates the response of the subject, associating it with each test.

- Sensor Agent: This role is taken on by as many agents as necessary. Their role in all cases is to take an appropriate action and communicate it to the rest of the system. In the present case, there are three agents which are related to the sensors responsible for monitoring the reactions of the subject of study.

- Classifier Agent: The task of the agents who implement this role consists of providing 
knowledge for the classification of information through AI techniques.

\section{Monitoring and control software}

This software is responsible for providing each user in the system with relevant information. Furthermore, it offers diverse options which enable users to interact with the system, as well as control and manage its different aspects.

Firstly, this software allows each user to access the system in a secure way (password identification) and and subscribes them to all the information flows that they should receive. Thus, asynchronously is received and displayed without the need for the user to need to sound the state of the system. At the same time, each user will be able to access all the information on the state of the machine from his device.

Secondly, the user is provided with an access point to the machine in the form of a display of options to execute. Thus, any information related to the action (or sequence of actions) to be performed is transmitted by publishing it in a module which is responsible for performing the server tasks for the exchange of information.

\section{CONCLUSION AND FUTURE RESEARCH LINES}

The development achieved in this work is the presented architecture that is capable of acting cognitively on multiple complex objects. With this architecture, end users can monitor and control objects from smart devices connected to the Internet; the function of the object used in the conducted case study was to examine the behaviour of animals in the presence of odours.

However, the proposed architecture is not limited to the object used in the case study but opens up many possibilities and can be applied to diverse problems. As a result, it opens new lines of research.

However, this device is sophisticated, being adapted to this architecture, can be monitored and controlled in real time from any device.

In addition, independently of the place in which they are located, all users who are connected to the machine will be able to view their status in real time and display current actions executed by other users present in the system.

Along with this multiuser feature, there is also a multimachine system, capable of controlling and displaying the status of different machines from the same interface. This is achieved by configuring the number of machines that are to be monitored.

By adapting novel technologies, such as IoT and WoT, the proposed architecture makes the work of researchers easier. It is not necessary anymore to connect computers physically to old research machines which can cause possible delays in communication, affecting their results. This cannot occur with the proposed architecture since the logical system is completely isolated from communications.

\section{REFERENCES}

[1] Xia, F., Yang, L. T., Wang, L., \& Vinel, A. (2012). Internet of things. International Journal of Communication Systems, 25(9), 1101

[2] Guinard, D., Trifa, V., Mattern, F., \& Wilde, E. (2011). From the internet of things to the web of things: Resource-oriented architecture and best practices. In Architecting the Internet of Things (pp. 97-129). Springer Berlin Heidelberg.

[3] Van der Hoek, W., \& Wooldridge, M. (2008). Multi-agent systems. Foundations of Artificial Intelligence, 3, 887-928.

[4] Atzori, L., Iera, A., \& Morabito, G. (2010). The internet of things: A survey. Computer networks, 54(15), 2787-2805.

[5] Chaouchi, H. (Ed.). (2013). The internet of things: connecting objects. John Wiley \& Sons.

[6] Chamoso, P., \& De La Prieta, F. (2015). Swarm-Based Smart City Platform: A Traffic Application. Advances in Distributed Computing and Artificial Intelligence Journal. Vol.4, n.2. (pp. 89-97). Ediciones Universidad de Salamanca.

[7] Mazhelis, O., Warma, H., Leminen, S., Ahokangas, P., Pussinen, P., Rajahonka, M., ... \& Myllykoski, J. (2013). Internet-of-things market, value networks, and business models: State of the art report. University of JYVÄSKYLÄ, DEPARTMENT OF COMPUTER SCIENCE AND INFORMATION SYSTEMS, Technical Reports TR-39, 13-14.

[8] Tozlu, S., Senel, M., Mao, W., \& Keshavarzian, A. (2012). Wi-Fi enabled sensors for internet of things: A practical approach. Communications Magazine, IEEE, 50(6), 134-143.

[9] An, L. (2012). Modeling human decisions in coupled human and natural systems: review of agent-based models. Ecological Modelling, 229, 2536.

[10] Van der Hoek, W., \& Wooldridge, M. (2008). Multi-agent systems. Foundations of Artificial Intelligence, 3, 887-928.

[11] Wooldridge, M., Jennings, N. R., \& Kinny, D. (2000). The Gaia methodology for agent-oriented analysis and design. Autonomous Agents and multi-agent systems, 3(3), 285-312.

[12] Ferber, J., Gutknecht, O., \& Michel, F. (2004). From agents to organizations: an organizational view of multi-agent systems. In AgentOriented Software Engineering IV (pp. 214-230). Springer Berlin Heidelberg. 